\title{
Infrared excess in semi-regular variables in the Magellanic Clouds
}

\author{
Martin A.T. Groenewegen ${ }^{1, \star}$ and Monika I. Jurkovic ${ }^{2,3, \star \star}$ \\ ${ }^{1}$ Koninklijke Sterrenwacht van België (KSB), Ringlaan 3, B-1180 Brussels, Belgium \\ ${ }^{2}$ Astronomical Observatory of Belgrade (AOB), Volgina 7, 11060 Belgrade, Serbia \\ ${ }^{3}$ Konkoly Observatory, Research Centre for Astronomy and Earth Sciences, Hungarian Academy of Sci- \\ ences, H-1121 Budapest, Konkoly Thege Miklós út 15-17, Hungary
}

\begin{abstract}
Following up on our recent work we are investigating if Type II Cepheids could be "hiding" in the sample of stars classified by the OGLE team as long-period variables.
\end{abstract}

\section{Introduction}

In two recent papers, [1, 2] (and see these proceedings, [3]) studied the 246 Type II Cepheids (T2Cs) and 89 Anomalous Cepheids (ANCEPs) discovered in the OGLE-III survey in the SMC and LMC $([5,7,8])$. T2C are pulsating stars associated with globular clusters and dwarf spheroidal galaxies and considered to be useful distance indicators for objects where classical Cepheids are too few and RR Lyrae too faint. They are typically separated into 3 subgroups according to their pulsation periods: the BL Herculis (BLH) stars have periods $1-4$ days, the W Virginis (WVir) $4-20$ days and the RV Tauris (RVT) 20 - 70 days pulsation periods. Variables with RVT-like light curves at longer periods may well exist but would appear in the OGLE samples of the long-period variables (LPVs) $([6,9])$.

In [1] the spectral energy distributions (SEDs) were constructed for all these stars, using available photometry from the literature, and fitted with a dust radiative transfer code. In the large majority of cases the SEDs could be fitted by a stellar photosphere model only. However, for $\sim 60 \%$ of the RVT and $\sim 10 \%$ of the (p)W Vir objects an infrared excess is detected from the SED fitting. The results of [4] are confirmed that there exist (pulsating) stars with luminosities below that predicted from single-star evolution that show a clear infrared excess, likely related to binarity.

In [2] we used the derived luminosities and effective temperatures to derive radii, and present period - (bolometric) luminosity, and period - radius relations for the T2C and ANCEP.

\section{T2C among LPVs/SRs}

In this (ongoing) study we want to look for T2C (mostly of the RVT type) in the OGLE catalogs of LPVs. The SMC and LMC sample of the regular survey were considered $([6,9])$ as well as the stars classified as LPVs in the OGLE Shallow Survey in the LMC ([10]) that contains brighter objects.

The initial selection was done on colour and magnitude, and required $I$-band amplitudes $>0.07$ mag.

\footnotetext{
${ }^{\star}$ martin.groenewegen@oma.be

$\star \star$ mojur@aob.rs
} 

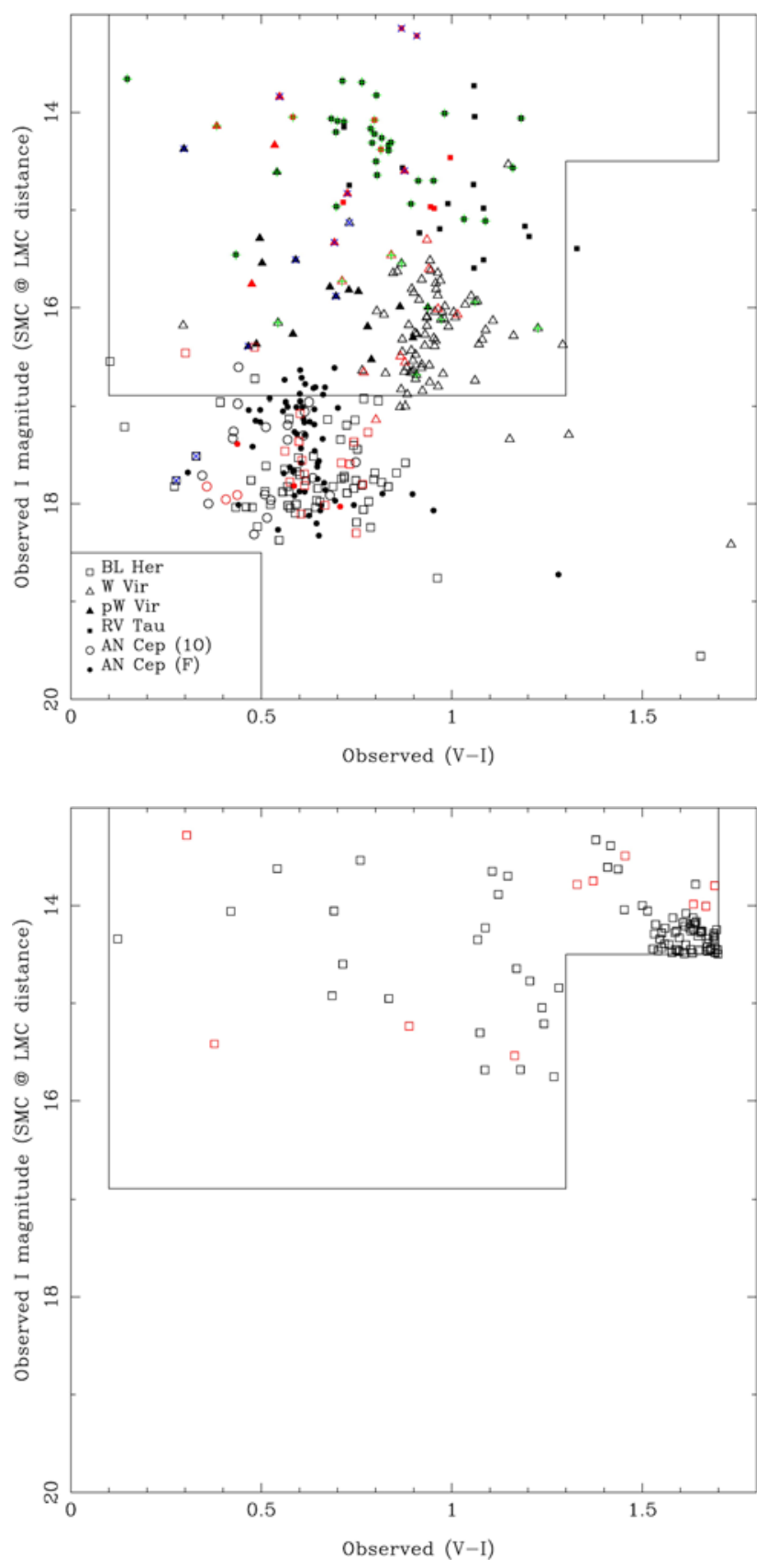

Figure 1. The figures show the observed $I,(V-I)$ CMD. Stars in the SMC are plotted in red, and shifted by -0.43 magnitudes. The top figure shows the sample of T2Cs and ANCEPs studied in $[1,2]$. Stars found to have an IR excess are marked by a green plus-sign. Stars which show eclipsing or ellipsoidal variations according to OGLE are marked by a blue cross. The rectangles indicates the colour box used in the present study. The bottom figure shows the stars selected from the OGLE LPV samples and the OGLE Shallow Survey. 

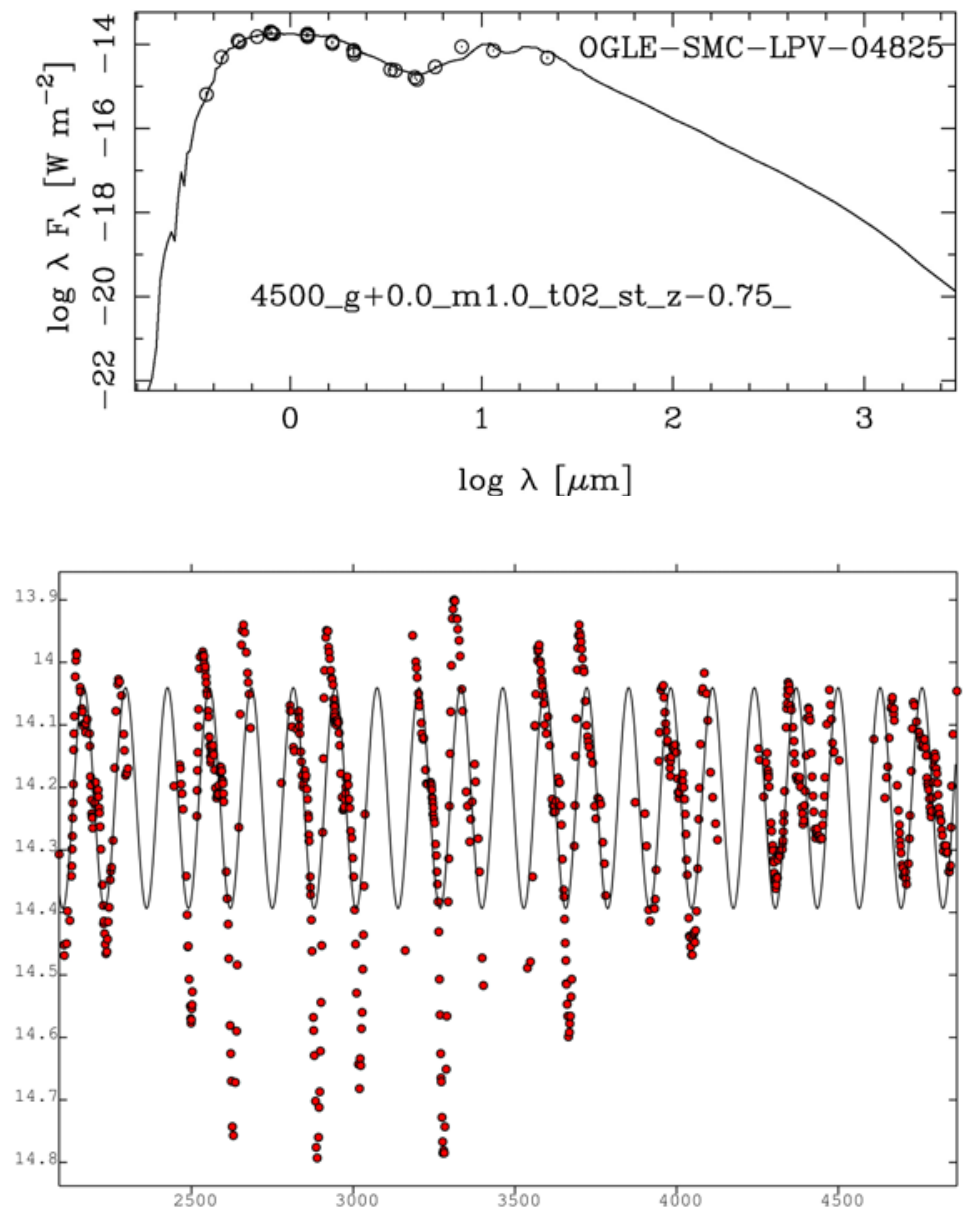

Figure 2. An example: The top panel shows a star with a clear IR excess. The bottom panel shows the OGLE light curve ( $I$-band magnitude versus time) of this object, and the best-fitting sine curve which has a period of 130 days.

The colour-magnitude diagram is shown in Figure 1. In the upper panel the selection box is shown together with the T2C and ANCEP sample of [1], and in the bottom panel the initial sample of 5 Miras, $78 \mathrm{SRV}, 35$ OSARGS, and 16 bright targets is displayed.

Phased light curves were constructed and inspected, also considering MACHO and EROS data when available. For the moment 29 stars have already been removed from the sample: 2 are also classified as T2C and are therefore already considered in [1], 13 are eclipsing binaries or show ellipsoidal variations, 3 of the bright stars have amplitudes smaller than the 0.07 magnitude limit, 3 have $\mathrm{R}$ CrB type light curves, and 8 have clear Mira like light curves. As this is an ongoing study, further reduction of the sample is very likely when considering light curve shape, period (range), luminosity (range), and the period-luminosity relation in more detail!

The SEDs were constructed and fitted as in [1]. Most stars can be fitted by a stellar photosphere alone, but other SEDs clearly show IR excess; see the example in Figure 2. 


\section{References}

[1] Groenewegen, M. A. T., \& Jurkovic, M. I., A\&A, in press (arXiv: 1705.00886) (2017a)

[2] Groenewegen, M. A. T., \& Jurkovic, M. I., A\&A, in press (arXiv:1705.04487) (2017b)

[3] Jurkovic, M. I., \& Groenewegen, M. A. T., these proceedings (2017)

[4] Kamath, D., Wood, P. R., Van Winckel, H., \& Nie, J. D., A\&A, 586, L5 (2016)

[5] Soszyński, I., Udalski, A., Szymański, M. K., et al., AcA, 58, 293 (2008)

[6] Soszyński, I., Udalski, A., Szymański, M. K., et al., AcA, 59, 239 (2009)

[7] Soszyński, I., Poleski, R., Udalski, A., et al., AcA, 60, 17 (2010)

[8] Soszyński, I., Udalski, A., Szymański, M. K., et al., AcA, 60, 91 (2010)

[9] Soszyński, I., Udalski, A., Szymański, M. K., et al., AcA, 61, 217 (2011)

[10] Ulaczyk, K., Szymański, M. K., Udalski, A., et al., AcA, 63, 159 (2013) 